\title{
Defining hypoglycaemia: what level has clinical relevance?
}

\author{
B. M. Frier
}

Received: 2 October 2008 / Accepted: 6 October 2008 / Published online: 19 November 2008

(C) Springer-Verlag 2008

Keywords Blood glucose $\cdot$ Counter-regulation $\cdot$ Diabetes . Glycaemic threshold $\cdot$ Hypoglycaemia $\cdot$ Impaired hypoglycaemia awareness $\cdot$ Insulin

\section{Abbreviation \\ ADA American Diabetes Association \\ Never let the facts get in the way of a carefully thought- out bad decision.}

John Marshall (1755-1835)

Because hypoglycaemia is so common in insulin-treated diabetes and remains the greatest impediment to strict glycaemic control, its evaluation is mandatory as an outcome measure of studies assessing new therapies for diabetes and those comparing insulin regimens or management strategies. Regrettably, the lack of consensus as to how hypoglycaemia should be defined has permitted, if not actively encouraged, a plethora of eccentric, vague and clinically irrelevant definitions to be applied in many therapeutic trials. The practical definition (from a clinical viewpoint) of severe (requiring external help for recovery) and mild (self-treated) hypoglycaemia, as described in the DCCT [1], has been widely adopted for epidemiological and clinical use, but its reliance on ascertaining the ability to self-treat hypoglycaemia does not capture events that do not generate symptoms - socalled asymptomatic 'biochemical' hypoglycaemia. This is a common problem in people with impaired awareness of hypoglycaemia, but is recognised to occur in all people treated with insulin, either because the glycaemic thresholds that trigger symptomatic and physiological responses are not broached, or warning symptoms are not subjectively per-

\section{B. M. Frier $(\bowtie)$}

Department of Diabetes, Royal Infirmary,

51 Little France Crescent,

Edinburgh EH16 4SA, UK

e-mail: brian.frier@luht.scot.nhs.uk ceived. The frequency with which biochemical hypoglycaemia appears to occur is dependent on how often it is measured. Estimates based on continuous glucose monitoring systems cannot be included because the sensors measure interstitial tissue glucose, and the inter-relationship between this and blood glucose is unclear.

\section{Rationale for the American Diabetes Association definition of biochemical hypoglycaemia}

A wide range of glucose concentrations could therefore represent biochemical hypoglycaemia; the difficulty arises in determining at what level it starts. To address this and other problems of defining hypoglycaemia, a Workgroup of the American Diabetes Association (ADA) published an erudite report in 2005 [2] in which the methods of identifying and measuring hypoglycaemia were reviewed and a classification of hypoglycaemia was proposed. Much of the report and recommendations are sound, sensible and justifiable, based on available evidence. The one contentious issue is their proposal that any blood glucose value equal to, or below, $3.9 \mathrm{mmol} / \mathrm{l}(70 \mathrm{mg} / \mathrm{dl})$ should represent hypoglycaemia, whether or not symptoms are present. Many clinicians would regard a blood glucose of this concentration in a non-diabetic adult as being within the normal fasting range, and would not consider it to indicate significant hypoglycaemia. This is an important consideration as it is evident that the glucose level that is selected to define hypoglycaemia can influence estimates of the frequency of this therapeutic side effect - a choice that could have important clinical and commercial implications. To illustrate this, Swinnen et al. [3] applied a range of different glucose levels defining hypoglycaemia to the blood glucose data from two large prospective trials of people with type 2 diabetes who had commenced treatment with insulin. As reported in this issue of Diabetologia, they unequivocally demonstrate how changing the glucose cut-off 
level can profoundly influence the estimated frequency of hypoglycaemia. The higher the cut-off level for hypoglycaemia, the greater the reported frequency, predominantly of asymptomatic events. The sensitivity to identify severe hypoglycaemia was not greatly enhanced by setting the definition of hypoglycaemia at a higher blood glucose level, but the specificity was lowered considerably. The authors suggest that exposure to blood glucose levels between 3.5 and $3.9 \mathrm{mmol} / \mathrm{l}$ is unlikely to be of great clinical consequence and argue that the ADA definition of $3.9 \mathrm{mmol} / \mathrm{l}$ is set at too high a level to have clinical credibility.

It is timely therefore to re-examine the ADA definition with respect to this designated blood glucose level of $3.9 \mathrm{mmol} / \mathrm{l}$ [2]. Why was this level chosen? The ADA Workgroup argued that this is the glycaemic level at which hormonal counter-regulation is activated in non-diabetic adults $[4,5]$, and that antecedent hypoglycaemia of this modest degree can reduce the secretion of the counterregulatory hormones glucagon and adrenaline (epinephrine) in response to a subsequent episode of hypoglycaemia [6].

\section{Type of blood sample used for glucose measurement}

It is important to appreciate that these key studies were all performed using the hyperinsulinaemic glucose clamp technique, in which plasma glucose is measured from samples of arterialised venous blood. This differs from the measurement of glucose in capillary whole blood, which is used routinely with most glucose meters in clinical practice. When ordinary venous blood is obtained by venepuncture in hospital practice, the laboratory usually measures plasma glucose, although this is not invariable. The plasma glucose concentration derived from arterialised venous blood is higher (about $15 \%$ ) than glucose measured in capillary or venous whole blood [7]. In the hypoglycaemic range the arteriovenous difference is small (probably less than $0.2 \mathrm{mmol} / \mathrm{l}$ ), but it can be influenced considerably by the level and duration of hyperinsulinaemia [8]. However, arterialised venous blood is not used for glucose measurements in therapeutic trials or for routine management, where capillary blood is the usual source for samples. It would seem more pertinent to define biochemical hypoglycaemia by measuring glucose in the type of blood sample that is typically used in clinical practice.

In addition, induction of hypoglycaemia using a glucose clamp technique is highly unphysiological and does not simulate how hypoglycaemia occurs in people treated with insulin, in whom blood glucose falls continuously and is not held at a plateau for predetermined intervals. In real life, when the blood glucose level reaches the threshold for sympathoadrenal activation, it triggers a profound 'autonomic reaction', a valuable descriptive term that has unfortunate- ly fallen into disuse, but which illustrates the sudden onset of symptomatic hypoglycaemia. Such an effect does not occur during a glucose clamp because of the slower method of lowering blood glucose (often in a stepwise manner) so that the onset of physiological changes and symptoms is gradual and progressive.

\section{Onset of counter-regulation for hypoglycaemia}

It is misleading to infer that the 'onset of counter-regulation' [2] is a sudden all-or-none phenomenon or that glycaemic thresholds for counter-regulatory secretion can be measured with precision. Striking discrepancies between the results of studies of the glycaemic thresholds for activation of counterregulatory hormones during hypoglycaemia have been reported, and Heine has discussed in detail the difficulties of accurate measurement by this method [9]. The reproducibility of the glycaemic thresholds was studied in normal individuals by Vea et al. [10], who showed mean intraindividual differences of $0.22 \mathrm{mmol} / \mathrm{l}$ for the secretion of counter-regulatory hormones and $0.25 \mathrm{mmol} / 1$ for the onset of symptoms. At best, only a range of glucose values can be identified within which hormone production increases by two standard deviations above the baseline level— the means by which a significant rise in hormonal secretion is defined in clamp studies. Not only do glycaemic thresholds differ between non-diabetic individuals, but accurate determination of the thresholds is difficult in all individuals, irrespective of whether or not they have insulin-treated diabetes. In addition, these glycaemic thresholds are dynamic in people with diabetes and can be influenced by many factors, such as the quality of glycaemic control or the development of impaired hypoglycaemia awareness [11]. It is debatable whether modest secretion of counter-regulatory hormones at blood glucose levels between 3.5 and $4.0 \mathrm{mmol} / \mathrm{l}$ has pathological significance, and in normal health may simply represent a glucose homeostatic mechanism that maintains glucose within a narrow physiological range. The relevance of basing a biochemical definition for hypoglycaemia on the glycaemic threshold for secretion of glucagon can also be disputed, as this becomes deficient at an early stage of type 1 diabetes [12] and in type 2 diabetes when pancreatic beta cell failure is present [13]. It therefore has no role in promoting glucose counter-regulation in people with insulin-treated diabetes.

\section{Antecedent hypoglycaemia and glycaemic thresholds for responses to hypoglycaemia}

Antecedent hypoglycaemia and recurrent exposure to hypoglycaemia are recognised causal mechanisms underly- 
ing the acquired conditions of impaired awareness of hypoglycaemia and counter-regulatory hormonal deficiency. These are components of the syndrome known as hypoglycaemia-associated autonomic failure (HAAF) [14]. This syndrome increases in prevalence with duration of type 1 diabetes. Exposure to antecedent hypoglycaemia diminishes symptomatic and counter-regulatory hormonal responses to subsequent hypoglycaemia [11]. However, most studies demonstrating these effects have subjected volunteers to antecedent hypoglycaemia using a glucose clamp for around $60 \mathrm{~min}$, a duration which may certainly occur during sleep, but would be unusual during waking hours. Repeated, short episodes of hypoglycaemia (at an arterialised venous blood glucose level of $2.8 \mathrm{mmol} / \mathrm{l}$ ) of 15 min duration in non-diabetic men, induced by intravenous injection of insulin, had no effect on counterregulatory hormones or symptomatic responses [15]. These results are in contrast to those from another glucose clamp study, which reported that a mere 5 min of antecedent hypoglycaemia (at an arterialised venous blood glucose level of $2.9 \mathrm{mmol} / \mathrm{l}$ ) influenced subsequent counter-regulatory responses to hypoglycaemia, but not symptoms [16]. It is possible that the method of induction of hypoglycaemia influences the magnitude of the effect of antecedent hypoglycaemia, but if this is so, then further studies of hypoglycaemia should be performed in which blood glucose is lowered by administering insulin by subcutaneous injection, in keeping with the usual method of delivery of insulin in clinical practice. The key study cited by the ADA Workgroup as providing evidence for a definitive effect of antecedent hypoglycaemia at a plasma glucose of $3.9 \mathrm{mmol} / \mathrm{l}$, again derived from arterialised venous blood, involved observations in eight young non-diabetic adults, whose symptom responses were not measured [6]. An effect on the glucagon and epinephrine responses to subsequent hypoglycaemia was described [6], but the significance of these findings to diabetes is unknown and they have not yet been replicated.

What is the clinician to make of these reported effects of antecedent hypoglycaemia in non-diabetic volunteers, conducted in the artificial environment of a research laboratory using non-physiological techniques? Are they relevant to the hypoglycaemia that occurs in people with insulin-treated diabetes in everyday life? A biochemical definition of hypoglycaemia that is based on the results of a solitary study of antecedent hypoglycaemia in nondiabetic adults is not compelling. While patient-orientated slogans such as 'Make Four the Floor' (Diabetes UK) are of practical value in encouraging safety and caution in users of insulin to avoid hypoglycaemia, few physicians will be convinced by the assertion that an arterialised glucose level of $3.9 \mathrm{mmol} / \mathrm{l}$ might herald the onset of significant hypoglycaemia.
Which blood glucose level defines clinically meaningful hypoglycaemia?

So what definition of biochemical hypoglycaemia would have greater clinical relevance? In non-diabetic volunteers and in people with type 1 diabetes who have normal awareness of hypoglycaemia and reasonable glycaemic control, symptomatic responses are not usually generated, and cognitive dysfunction does not commence, until the (arterialised venous) blood glucose level approaches $3.2 \mathrm{mmol} / 1$ [11]. An arbitrary cut-off level of $3.5 \mathrm{mmol} / 1$ to define the onset of hypoglycaemia in clinical practice would probably be acceptable to most clinicians. It is still above the accepted glycaemic thresholds for the onset of the manifestations of hypoglycaemia that result from the stimulation of the sympathoadrenal system and the onset of cognitive dysfunction, and it is the activation of these responses that generate autonomic and neuroglycopenic warning symptoms. Although these responses may be preceded by some release of counter-regulatory hormones in non-diabetic individuals, the absence of glucagon secretion in insulin-deficient diabetes negates the clinical importance of defining hypoglycaemia on this basis. While the difference between 3.5 and $3.9 \mathrm{mmol} / \mathrm{l}$ may appear to be insignificant, Swinnen et al. [3] have clearly illustrated how a small change in the cut-off level can magnify the prevalence of asymptomatic hypoglycaemia. Ironically, an arterialised venous glucose level of $3.9 \mathrm{mmol} / 1$ is probably equivalent to a capillary blood glucose level of $3.5 \mathrm{mmol} / \mathrm{l}$. By defining biochemical hypoglycaemia as a capillary blood glucose level of $3.5 \mathrm{mmol} / \mathrm{l}$ or lower, the unnecessary inflation of the frequency of clinically meaningless biochemical hypoglycaemia in clinical studies could be avoided.

Duality of interest The author declares that there is no duality of interest associated with this manuscript.

\section{References}

1. The Diabetes Control and Complications Trial Research Group (1993) The effect of intensive insulin treatment of diabetes on the development and progression of long-term complications in insulin-dependent diabetes mellitus. N Engl J Med 329:977986

2. American Diabetes Association Workgroup on Hypoglycemia (2005) Defining and reporting hypoglycemia in diabetes: a report from the American Diabetes Association Workgroup on Hypoglycemia. Diabetes Care 28:1245-1249

3. Swinnen SGHA, Mullins P, Miller M, Hoekstra JBL, Holleman F (2009) Changing the glucose cut-off values that define hypoglycaemia has a major effect on reported frequencies of hypoglycaemia. Diabetologia doi:10.1007/s00125-008-1147-0 
4. Schwartz NS, Clutter WE, Shah SD, Cryer PE (1987) Glycemic thresholds for activation of glucose counterregulatory systems are higher than the threshold for symptoms. J Clin Invest 79:777-781

5. Mitrakou A, Ryan C, Veneman T et al (1991) Hierarchy of glycemic thresholds for counterregulatory hormone secretion, symptoms, and cerebral dysfunction. Am J Physiol Endocrinol Metab 260:E67-E74

6. Davis SN, Shavers C, Mosqueda-Garcia R, Costa F (1997) Effects of differing antecedent hypoglycemia on subsequent counterregulation in normal humans. Diabetes 46:1328-1335

7. Liu D, Moberg E, Kollind M, Lins P-E, Adamson U, Macdonald IA (1992) Arterial, arterialized venous, venous and capillary blood glucose measurements in normal man during hyperinsulinaemic euglycaemia and hypoglycaemia. Diabetologia 35:287-290

8. Liu D, Moberg E, Kollind M, Lins P-E, Adamson U (1991) A high concentration of circulating insulin suppresses the glucagon response to hypoglycemia in normal man. J Clin Endocrinol Metab 73:1123-1128

9. Heine RJ (1993) Methods of investigation of insulin-induced hypoglycaemia. In: Frier BM, Fisher BM (eds) Hypoglycaemia and diabetes: clinical and physiological aspects. Edward Arnold, London, pp 165-175
10. Vea H, Jorde R, Sager G, Vaaler S, Sundsfjord J (1992) Reproducibility of glycaemic thresholds for activation of counterregulatory hormones and hypoglycaemic symptoms in healthy subjects. Diabetologia 35:958-961

11. Frier BM (2007) Impaired awareness of hypoglycaemia. In: Frier BM, Fisher M (eds) Hypoglycaemia in clinical diabetes, 2nd edn. Wiley, Chichester, pp 141-170

12. Bolli GB, De Feo P, Campagnucci P et al (1983) Abnormal glucose counterregulation in insulin-dependent diabetes mellitus. Interaction of anti-insulin antibodies and impaired glucagon and epinephrine secretion. Diabetes 32:134-141

13. Segel SA, Paramore DA, Cryer PE (2002) Hypoglycemiaassociated autonomic failure in advanced type 2 diabetes. Diabetes 51:724-733

14. Cryer PE (2002) Hypoglycaemia: the limiting factor in the management of type 1 and type 2 diabetes. Diabetologia 45:937-948

15. Peters A, Rohloff F, Kerner W (1995) Preserved counterregulatory hormone release and symptoms after short term hypoglycemic episodes in normal men. J Clin Endocrinol Metab 80:2894-2898

16. Davis SN, Mann S, Galassetti P et al (2000) Effects of differing durations of antecedent hypoglycemia on counterregulatory responses to subsequent hypoglycemia in normal humans. Diabetes 49:1897-1903 